\title{
Integral of Complex-Valued Measurable Function
}

\author{
Keiko Narita \\ Hirosaki-city \\ Aomori, Japan
}

\author{
Noboru Endou \\ Gifu National College of Technology \\ Japan \\ Yasunari Shidama \\ Shinshu University \\ Nagano, Japan
}

\begin{abstract}
Summary. In this article, we formalized the notion of the integral of a complex-valued function considered as a sum of its real and imaginary parts. Then we defined the measurability and integrability in this context, and proved the linearity and several other basic properties of complex-valued measurable functions. The set of properties showed in this paper is based on [15], where the case of real-valued measurable functions is considered.
\end{abstract}

MML identifier: MESFUN6C, version: $\underline{7.9 .01 \quad 4.101 .1015}$

The notation and terminology used here are introduced in the following papers: [17], [1], [11], [18], [6], [19], [7], [2], [12], [14], [16], [5], [4], [3], [9], [10], [13], [8], and $[15]$.

\section{Definitions for Complex-Valued Functions}

One can prove the following proposition

(1) For all real numbers $a, b$ holds $\overline{\mathbb{R}}(a)+\overline{\mathbb{R}}(b)=a+b$ and $-\overline{\mathbb{R}}(a)=-a$ and $\overline{\mathbb{R}}(a)-\overline{\mathbb{R}}(b)=a-b$ and $\overline{\mathbb{R}}(a) \cdot \overline{\mathbb{R}}(b)=a \cdot b$.

Let $X$ be a non empty set and let $f$ be a partial function from $X$ to $\mathbb{C}$. The functor $\Re(f)$ yields a partial function from $X$ to $\mathbb{R}$ and is defined as follows:

(Def. 1) $\operatorname{dom} \Re(f)=\operatorname{dom} f$ and for every element $x$ of $X$ such that $x \in \operatorname{dom} \Re(f)$ holds $\Re(f)(x)=\Re(f(x))$. 
The functor $\Im(f)$ yields a partial function from $X$ to $\mathbb{R}$ and is defined as follows:

(Def. 2) $\operatorname{dom} \Im(f)=\operatorname{dom} f$ and for every element $x$ of $X$ such that $x \in \operatorname{dom} \Im(f)$ holds $\Im(f)(x)=\Im(f(x))$.

\section{The Measurability of Complex-valued Functions}

For simplicity, we use the following convention: $X$ is a non empty set, $Y$ is a set, $S$ is a $\sigma$-field of subsets of $X, M$ is a $\sigma$-measure on $S, f, g$ are partial functions from $X$ to $\mathbb{C}, r$ is a real number, $c$ is a complex number, and $E, A, B$ are elements of $S$.

Let $X$ be a non empty set, let $S$ be a $\sigma$-field of subsets of $X$, let $f$ be a partial function from $X$ to $\mathbb{C}$, and let $E$ be an element of $S$. We say that $f$ is measurable on $E$ if and only if:

(Def. 3) $\Re(f)$ is measurable on $E$ and $\Im(f)$ is measurable on $E$.

One can prove the following propositions:

(2) $\quad r \Re(f)=\Re(r f)$ and $r \Im(f)=\Im(r f)$.

(3) $\Re(c f)=\Re(c) \Re(f)-\Im(c) \Im(f)$ and $\Im(c f)=\Im(c) \Re(f)+\Re(c) \Im(f)$.

(4) $-\Im(f)=\Re(i f)$ and $\Re(f)=\Im(i f)$.

(5) $\Re(f+g)=\Re(f)+\Re(g)$ and $\Im(f+g)=\Im(f)+\Im(g)$.

(6) $\Re(f-g)=\Re(f)-\Re(g)$ and $\Im(f-g)=\Im(f)-\Im(g)$.

(7) $\Re(f)\lceil A=\Re(f\lceil A)$ and $\Im(f)\lceil A=\Im(f\lceil A)$.

(8) $f=\Re(f)+i \Im(f)$.

(9) If $B \subseteq A$ and $f$ is measurable on $A$, then $f$ is measurable on $B$.

(10) If $f$ is measurable on $A$ and $f$ is measurable on $B$, then $f$ is measurable on $A \cup B$.

(11) If $f$ is measurable on $A$ and $g$ is measurable on $A$, then $f+g$ is measurable on $A$.

(12) If $f$ is measurable on $A$ and $g$ is measurable on $A$ and $A \subseteq \operatorname{dom} g$, then $f-g$ is measurable on $A$.

(13) If $Y \subseteq \operatorname{dom}(f+g)$, then $\operatorname{dom}(f\lceil Y+g \uparrow Y)=Y$ and $(f+g) \uparrow Y=f \uparrow Y+g \uparrow Y$.

(14) If $f$ is measurable on $B$ and $A=\operatorname{dom} f \cap B$, then $f\lceil B$ is measurable on $A$.

(15) If $\operatorname{dom} f, \operatorname{dom} g \in S$, then $\operatorname{dom}(f+g) \in S$.

(16) If $\operatorname{dom} f=A$, then $f$ is measurable on $B$ iff $f$ is measurable on $A \cap B$.

(17) If $f$ is measurable on $A$ and $A \subseteq \operatorname{dom} f$, then $c f$ is measurable on $A$.

(18) Given an element $A$ of $S$ such that $\operatorname{dom} f=A$. Let $c$ be a complex number and $B$ be an element of $S$. If $f$ is measurable on $B$, then $c f$ is measurable on $B$. 


\section{The Integral of a Complex-valued Measurable Function}

Let $X$ be a non empty set, let $S$ be a $\sigma$-field of subsets of $X$, let $M$ be a $\sigma$-measure on $S$, and let $f$ be a partial function from $X$ to $\mathbb{C}$. We say that $f$ is integrable on $M$ if and only if:

(Def. 4) $\Re(f)$ is integrable on $M$ and $\Im(f)$ is integrable on $M$.

Let $X$ be a non empty set, let $S$ be a $\sigma$-field of subsets of $X$, let $M$ be a $\sigma$-measure on $S$, and let $f$ be a partial function from $X$ to $\mathbb{C}$. Let us assume that $f$ is integrable on $M$. The functor $\int f \mathrm{~d} M$ yielding a complex number is defined by:

(Def. 5) There exist real numbers $R, I$ such that $R=\int \Re(f) \mathrm{d} M$ and $I=$ $\int \Im(f) \mathrm{d} M$ and $\int f \mathrm{~d} M=R+I \cdot i$.

We now state several propositions:

(19) Let $X$ be a non empty set, $S$ be a $\sigma$-field of subsets of $X, M$ be a $\sigma$ measure on $S, f$ be a partial function from $X$ to $\overline{\mathbb{R}}$, and $A$ be an element of $S$. Suppose there exists an element $E$ of $S$ such that $E=\operatorname{dom} f$ and $f$ is measurable on $E$ and $M(A)=0$. Then $f\lceil A$ is integrable on $M$.

(20) Let $X$ be a non empty set, $S$ be a $\sigma$-field of subsets of $X, M$ be a $\sigma$ measure on $S, f$ be a partial function from $X$ to $\mathbb{R}$, and $E, A$ be elements of $S$. Suppose there exists an element $E$ of $S$ such that $E=\operatorname{dom} f$ and $f$ is measurable on $E$ and $M(A)=0$. Then $f \nmid A$ is integrable on $M$.

(21) Suppose there exists an element $E$ of $S$ such that $E=\operatorname{dom} f$ and $f$ is measurable on $E$ and $M(A)=0$. Then $f \nmid A$ is integrable on $M$ and $\int f\lceil A \mathrm{~d} M=0$.

(22) If $E=\operatorname{dom} f$ and $f$ is integrable on $M$ and $M(A)=0$, then $\int f \uparrow(E \backslash$ A) $\mathrm{d} M=\int f \mathrm{~d} M$.

(23) If $f$ is integrable on $M$, then $f \uparrow A$ is integrable on $M$.

(24) If $f$ is integrable on $M$ and $A$ misses $B$, then $\int f \uparrow(A \cup B) \mathrm{d} M=$ $\int f\left\lceil A \mathrm{~d} M+\int f\lceil B \mathrm{~d} M\right.$.

(25) If $f$ is integrable on $M$ and $B=\operatorname{dom} f \backslash A$, then $f\lceil A$ is integrable on $M$ and $\int f \mathrm{~d} M=\int f\left\lceil A \mathrm{~d} M+\int f\lceil B \mathrm{~d} M\right.$.

Let $k$ be a real number, let $X$ be a non empty set, and let $f$ be a partial function from $X$ to $\mathbb{R}$. The functor $f^{k}$ yields a partial function from $X$ to $\mathbb{R}$ and is defined as follows:

(Def. 6) $\operatorname{dom}\left(f^{k}\right)=\operatorname{dom} f$ and for every element $x$ of $X$ such that $x \in \operatorname{dom}\left(f^{k}\right)$ holds $f^{k}(x)=f(x)^{k}$.

Let us consider $X$. Observe that there exists a partial function from $X$ to $\mathbb{R}$ which is non-negative.

Let $k$ be a non negative real number, let us consider $X$, and let $f$ be a non-negative partial function from $X$ to $\mathbb{R}$. Observe that $f^{k}$ is non-negative. 
We now state a number of propositions:

(26) Let $k$ be a real number, given $X, S, E$, and $f$ be a partial function from $X$ to $\mathbb{R}$. If $f$ is non-negative and $0 \leq k$, then $f^{k}$ is non-negative.

(27) Let $x$ be a set, given $X, S, E$, and $f$ be a partial function from $X$ to $\mathbb{R}$. If $f$ is non-negative, then $f(x)^{\frac{1}{2}}=\sqrt{f(x)}$.

(28) For every partial function $f$ from $X$ to $\mathbb{R}$ and for every real number $a$ such that $A \subseteq \operatorname{dom} f$ holds $A \cap \operatorname{LE}-\operatorname{dom}(f, a)=A \backslash A \cap \mathrm{GTE}-\operatorname{dom}(f, a)$.

(29) Let $k$ be a real number, given $X, S, E$, and $f$ be a partial function from $X$ to $\mathbb{R}$. Suppose $f$ is non-negative and $0 \leq k$ and $E \subseteq \operatorname{dom} f$ and $f$ is measurable on $E$. Then $f^{k}$ is measurable on $E$.

(30) If $f$ is measurable on $A$ and $A \subseteq \operatorname{dom} f$, then $|f|$ is measurable on $A$.

(31) Given an element $A$ of $S$ such that $A=\operatorname{dom} f$ and $f$ is measurable on $A$. Then $f$ is integrable on $M$ if and only if $|f|$ is integrable on $M$.

(32) If $f$ is integrable on $M$ and $g$ is integrable on $M$, then $\operatorname{dom}(f+g) \in S$.

(33) If $f$ is integrable on $M$ and $g$ is integrable on $M$, then $f+g$ is integrable on $M$.

(34) Let $X$ be a non empty set, $S$ be a $\sigma$-field of subsets of $X, M$ be a $\sigma$ measure on $S$, and $f, g$ be partial functions from $X$ to $\mathbb{R}$. Suppose $f$ is integrable on $M$ and $g$ is integrable on $M$. Then $f-g$ is integrable on $M$.

(35) If $f$ is integrable on $M$ and $g$ is integrable on $M$, then $f-g$ is integrable on $M$.

(36) Suppose $f$ is integrable on $M$ and $g$ is integrable on $M$. Then there exists an element $E$ of $S$ such that $E=\operatorname{dom} f \cap \operatorname{dom} g$ and $\int f+g \mathrm{~d} M=$ $\int f\left\lceil E \mathrm{~d} M+\int g\lceil E \mathrm{~d} M\right.$.

(37) Let $X$ be a non empty set, $S$ be a $\sigma$-field of subsets of $X, M$ be a $\sigma$ measure on $S$, and $f, g$ be partial functions from $X$ to $\mathbb{R}$. Suppose $f$ is integrable on $M$ and $g$ is integrable on $M$. Then there exists an element $E$ of $S$ such that $E=\operatorname{dom} f \cap \operatorname{dom} g$ and $\int f-g \mathrm{~d} M=\int f\lceil E \mathrm{~d} M+$ $\int(-g)\lceil E \mathrm{~d} M$.

(38) If $f$ is integrable on $M$, then $r f$ is integrable on $M$ and $\int r f \mathrm{~d} M=$ $r \cdot \int f \mathrm{~d} M$.

(39) If $f$ is integrable on $M$, then if is integrable on $M$ and $\int i f \mathrm{~d} M=$ $i \cdot \int f \mathrm{~d} M$.

(40) If $f$ is integrable on $M$, then $c f$ is integrable on $M$ and $\int c f \mathrm{~d} M=$ $c \cdot \int f \mathrm{~d} M$.

(41) For every partial function $f$ from $X$ to $\mathbb{R}$ and for all $Y, r$ holds $(r f) \mid Y=$ $r(f \nmid Y)$.

(42) Let $f, g$ be partial functions from $X$ to $\mathbb{R}$. Suppose that 
(i) there exists an element $A$ of $S$ such that $A=\operatorname{dom} f \cap \operatorname{dom} g$ and $f$ is measurable on $A$ and $g$ is measurable on $A$,

(ii) $f$ is integrable on $M$,

(iii) $g$ is integrable on $M$, and

(iv) $g-f$ is non-negative.

Then there exists an element $E$ of $S$ such that $E=\operatorname{dom} f \cap \operatorname{dom} g$ and $\int f\left\lceil E \mathrm{~d} M \leq \int g\lceil E \mathrm{~d} M\right.$.

(43) Suppose there exists an element $A$ of $S$ such that $A=\operatorname{dom} f$ and $f$ is measurable on $A$ and $f$ is integrable on $M$. Then $\left|\int f \mathrm{~d} M\right| \leq \int|f| \mathrm{d} M$.

Let $X$ be a non empty set, let $S$ be a $\sigma$-field of subsets of $X$, let $M$ be a $\sigma$-measure on $S$, let $f$ be a partial function from $X$ to $\mathbb{C}$, and let $B$ be an element of $S$. The functor $\int_{B} f \mathrm{~d} M$ yields a complex number and is defined by: (Def. 7) $\int_{B} f \mathrm{~d} M=\int f\lceil B \mathrm{~d} M$.

Next we state two propositions:

(44) Suppose $f$ is integrable on $M$ and $g$ is integrable on $M$ and $B \subseteq \operatorname{dom}(f+$ $g$ ). Then $f+g$ is integrable on $M$ and $\int_{B} f+g \mathrm{~d} M=\int_{B} f \mathrm{~d} M+\int_{B} g \mathrm{~d} M$.

(45) If $f$ is integrable on $M$ and $f$ is measurable on $B$, then $\int_{B} c f \mathrm{~d} M=$ $c \cdot \int_{B} f \mathrm{~d} M$.

\section{Several Properties of Real-valued Measurable Functions}

In the sequel $f$ denotes a partial function from $X$ to $\mathbb{R}$ and $a$ denotes a real number.

One can prove the following four propositions:

(46) If $A \subseteq \operatorname{dom} f$, then $A \cap \mathrm{GTE}-\operatorname{dom}(f, a)=A \backslash A \cap \operatorname{LE}-\operatorname{dom}(f, a)$.

(47) If $A \subseteq \operatorname{dom} f$, then $A \cap \mathrm{GT}-\operatorname{dom}(f, a)=A \backslash A \cap \operatorname{LEQ-dom}(f, a)$.

(48) If $A \subseteq \operatorname{dom} f$, then $A \cap \mathrm{LEQ}-\operatorname{dom}(f, a)=A \backslash A \cap \mathrm{GT}-\operatorname{dom}(f, a)$.

(49) $A \cap \mathrm{EQ}-\operatorname{dom}(f, a)=A \cap \operatorname{GTE}-\operatorname{dom}(f, a) \cap \operatorname{LEQ}-\operatorname{dom}(f, a)$.

\section{REFERENCES}

[1] Grzegorz Bancerek. The ordinal numbers. Formalized Mathematics, 1(1):91-96, 1990.

[2] Józef Białas. Series of positive real numbers. Measure theory. Formalized Mathematics, 2(1):173-183, 1991

[3] Józef Białas. The $\sigma$-additive measure theory. Formalized Mathematics, 2(2):263-270, 1991.

[4] Józef Białas. Some properties of the intervals. Formalized Mathematics, 5(1):21-26, 1996.

[5] Czesław Byliński. The complex numbers. Formalized Mathematics, 1(3):507-513, 1990.

[6] Czesław Byliński. Functions and their basic properties. Formalized Mathematics, 1(1):55$65,1990$.

[7] Czesław Byliński. Partial functions. Formalized Mathematics, 1(2):357-367, 1990. 
[8] Noboru Endou and Yasunari Shidama. Integral of measurable function. Formalized Mathematics, 14(2):53-70, 2006.

[9] Noboru Endou, Katsumi Wasaki, and Yasunari Shidama. Basic properties of extended real numbers. Formalized Mathematics, 9(3):491-494, 2001.

[10] Noboru Endou, Katsumi Wasaki, and Yasunari Shidama. Definitions and basic properties of measurable functions. Formalized Mathematics, 9(3):495-500, 2001.

[11] Krzysztof Hryniewiecki. Basic properties of real numbers. Formalized Mathematics, 1(1):35-40, 1990.

[12] Jarosław Kotowicz. Real sequences and basic operations on them. Formalized Mathematics, 1(2):269-272, 1990.

[13] Andrzej Nędzusiak. $\sigma$-fields and probability. Formalized Mathematics, 1(2):401-407, 1990.

[14] Konrad Raczkowski and Andrzej Nȩdzusiak. Real exponents and logarithms. Formalized Mathematics, 2(2):213-216, 1991.

[15] Yasunari Shidama and Noboru Endou. Integral of real-valued measurable function. Formalized Mathematics, 14(4):143-152, 2006.

[16] Andrzej Trybulec and Czesław Byliński. Some properties of real numbers. Formalized Mathematics, 1(3):445-449, 1990.

[17] Zinaida Trybulec. Properties of subsets. Formalized Mathematics, 1(1):67-71, 1990.

[18] Edmund Woronowicz. Relations and their basic properties. Formalized Mathematics, 1(1):73-83, 1990.

[19] Edmund Woronowicz. Relations defined on sets. Formalized Mathematics, 1(1):181-186, 1990. 